The Astrophysical Journal, 625:688-698, 2005 June 1

(C) 2005. The American Astronomical Society. All rights reserved. Printed in U.S.A.

\title{
VARIABILITY OF Fe II EMISSION FEATURES IN THE SEYFERT 1 GALAXY NGC 5548
}

\author{
Marianne Vestergaard ${ }^{1,2}$ and Bradley M. Peterson ${ }^{1}$ \\ Received 2004 October 27; accepted 2005 February 17
}

\begin{abstract}
We study the low-contrast Fe II emission blends in the ultraviolet (1250-2200 $)$ and optical (4000-6000 $)$ spectra of the Seyfert 1 galaxy NGC 5548 and show that these features vary in flux and that these variations are correlated with those of the optical continuum. The amplitude of variability of the optical $\mathrm{Fe}$ II emission is $50 \%-$ $75 \%$ that of $\mathrm{H} \beta$, and the ultraviolet $\mathrm{Fe}$ II emission varies with an even larger amplitude than $\mathrm{H} \beta$. However, accurate measurement of the flux in these blends proves to be very difficult even using excellent Fe II templates to fit the spectra. We are able to constrain only weakly the optical Fe II emission-line response timescale to a value of less than several weeks; this upper limit exceeds all the reliably measured emission-line lags in this source so it is not particularly meaningful. Nevertheless, the fact that the optical $\mathrm{Fe}$ II and continuum flux variations are correlated indicates that line fluorescence in a photoionized plasma, rather than collisional excitation, is responsible for the Fe II emission. The iron emission templates are available upon request.
\end{abstract}

Subject headings: galaxies: active — galaxies: nuclei — galaxies: Seyfert — quasars: emission lines

\section{INTRODUCTION}

Emission from singly ionized iron is one of the principal coolants of the broad-line region (BLR) in active galactic nuclei (AGNs). Indeed, the prominent blend of Fe II and Balmer continuum emission in the near UV spectrum, a feature often referred to as the "small blue bump," produces as much as one-third of the line flux in some objects (Wills et al. 1985; Maoz et al. 1993). Remarkably, however, the characteristics of Fe II emission in AGNs are very poorly understood from both theoretical and observational perspectives:

1. The excitation mechanism that produces $\mathrm{Fe}$ II emission in AGN spectra is not known.-Theoretical models, based on either photoionization or collisional excitation, have for a long time fallen short of reproducing the observed $\mathrm{Fe}$ II emission (e.g., Collin \& Joly 2000; Baldwin et al. 2004). Also, conflicting results appear in the literature as to the importance of the hard X-rays as an excitation agent, as originally anticipated by Davidson \& Netzer (1979), and as to that of the slope of the soft X-ray spectrum (e.g., Wilkes et al. 1987; Shastri et al. 1994; Boroson 1989; Zheng \& O'Brien 1990; see also Wilkes et al. 1999). Even with much-improved current models, either significant microturbulence (or velocity shear) in a photoionized medium or a contribution from a collisionally ionized gas seems to be required to account for the strength and shape of $\mathrm{Fe}$ II emission in AGN spectra (e.g., Baldwin et al. 2004). The conditions that are necessary to reproduce the observed Fe II spectra do not provide clear and straightforward diagnostics to distinguish between these possibilities.

2. Strong, broad $\mathrm{Fe}$ II emission pervades the UV through IR spectra of AGNs, contaminating other spectral features.-The complex atomic structure of $\mathrm{Fe}^{+}$results in tens of thousands of transitions (e.g., Verner et al. 1999; Sigut \& Pradhan 2003) that produce blended features across the UV, optical, and IR spectrum. Isolating individual emission lines under these circum-

\footnotetext{
1 Department of Astronomy, Ohio State University, 140 West 18th Avenue, Columbus, OH 43210; peterson@astronomy.ohio-state.edu.

2 Steward Observatory, University of Arizona, 933 North Cherry Avenue, Tucson, AZ 85721; mvestergaard@as.arizona.edu.
}

stances is challenging, leading to systematic uncertainties in measurement of emission-line fluxes, widths, and profiles. The intrinsically large line widths of AGN emission lines add to the complexity of this issue.

Further underscoring the importance of understanding AGN Fe II emission is the possibility of using AGN spectra to measure the cosmic evolution of the abundance of iron, thus providing potentially strong constraints on the history of chemical enrichment due to star formation (e.g., Hamann \& Ferland 1999; Dietrich et al. 2003 and references therein; but see also Verner et al. 2004; Baldwin et al. 2004).

A potentially powerful way of exploring the Fe II-emitting region in AGNs is through flux variability, specifically by emissionline reverberation mapping (Blandford \& McKee 1982; Peterson 1993, 2001). At minimum, reverberation mapping can be expected to yield the typical distance of the Fe II-emitting gas from the central continuum source through measurement of the timedelayed response of the emission lines to continuum variations. This would allow us to constrain observationally some of the physical conditions conducive to strong Fe II emission. For example, variability timescales for the optical and UV Fe II emission, respectively, will help us to discern whether or not the multiplets at these energies originate in a common region. This is a key issue for understanding their relative intensity ratio in terms of current $\mathrm{Fe}$ II models, which fail to reproduce observations (Baldwin et al. 2004). At the present time, studies of Fe II flux variability have been very limited:

1. Maoz et al. (1993) used UV data obtained with the International Ultraviolet Explorer (IUE) and optical data obtained with multiple ground-based telescopes by the International AGN Watch $^{3}$ over an 8 month period in 1989 to study the variability of the small blue bump in NGC 5548. They concluded that the small blue bump emission arises at approximately the same distance as the Ly $\alpha$ emission, about 10 lt-days from the continuum source. While in many ways this represents the best study to date

\footnotetext{
3 All International AGN Watch data are available at http://www.astronomy .ohio-state.edu/ agnwatch.
} 
on the variability of the small blue bump, it must be noted that (a) the quality of IUE data in this part of the spectrum (i.e., obtained with the LWP camera) is quite poor and (b) there are relatively few epochs in which there is no gap in wavelength coverage between the UV and optical spectra, making measurements of the small blue bump variability systematically somewhat uncertain.

2. Goad et al. (1999) observed the small blue bump in NGC 3516 on six occasions over an 11 month period in 1995-1996. Remarkably, while the continuum and high-ionization emission lines varied in flux by a factor of 2 , the $\mathrm{Mg}$ II $\lambda 2800$ and Fe II UV emission did not vary by more than $7 \%$ over this time.

3. Kollatschny et al. (2000) carried out a 20 year study of emission-line variations in NGC 7603 and found that the optical Fe II blends vary with an amplitude comparable to that of the Balmer lines.

4. Kollatschny \& Welsh (2001) searched for and found no optical Fe II variability in Mrk 110 to within their sensitivity of $10 \%$, although clear optical Fe II variability is seen in the case of Fairall 9 with an amplitude half that of $\mathrm{H} \beta$ (Kollatschny \& Fricke 1985).

5. Sergeev et al. (1997) attempted to constrain the time delay of the Fe II multiplets in the wings of [O III] $\lambda 5007$ in the spectrum of NGC 5548. They found a time delay of a few hundred days. There are, however, a number of serious difficulties with this result arising from strong blending of lines, data quality, and time series problems.

6. A few other studies make claims of Fe II variability (Kollatschny et al. 1981; Salamanca et al. 1995; Doroshenko et al. 1999; Wamsteker et al. 1990). On the basis of spectra of narrow-line Seyfert 1 galaxies taken a year apart, Giannuzzo \& Stirpe (1996) find that the optical Fe II does seem to vary in these sources. For the three sources with detected variability in both $\mathrm{H} \beta$ and $\mathrm{Fe}$ II, the $\mathrm{Fe}$ II $/ \mathrm{H} \beta$ flux ratio ranged from 0.25 to 1.0 ; this would put Fe II variability below the detection threshold for the sources in which it was not detected.

Given the importance of understanding $\mathrm{Fe}$ II emission in AGNs and given the limited attention this topic has received in the past, we have undertaken an examination of Fe II variability in NGC 5548. In terms of emission-line variability, NGC 5548 is by far the best-studied AGN (see Peterson et al. 2002 and references therein). On the other hand, as Baldwin et al. (2004) point out, Fe II emission is not especially strong in this source, and other sources may be better suited to such a study. While we agree with this statement, we also feel that the wealth of data on other lines in NGC 5548 affords a special context in which to examine Fe II variability, and, of course, suitable data for this study already exist. Moreover, cursory consideration of Fe II variability in NGC 5548 yields mixed impressions. For example, the difference between spectra taken at recent historical maximum and minimum flux states (see Peterson et al. 1999, Fig. 3) seems to clearly show optical Fe II emission, indicating that these blends do indeed vary in this source. However, Kollatschny \& Welsh (2001) attribute the apparent variations solely to changes in He II 24686. If indeed the optical Fe II emission varies with time, then we ought to see evidence of these features in the difference or rms spectra (defined below in $\S 2.3 .2$ ) that we use to isolate the variable parts of the emission lines (e.g., Peterson et al. 2004). Our initial impression is that the optical Fe II blends do not appear in rms spectra, and thus there is no compelling evidence for optical Fe II variability, at least on timescales of several months.

Thus, the goal of this study is to determine whether or not Fe II emission varies in NGC 5548 and, if so, with what amplitude and on what timescale. We also attempt to constrain meaningfully the Fe II line width as an additional test of the common assumption that the optical Fe II emission arises cospatially with the Balmer lines (e.g., Phillips 1978). We do not specifically reexamine the issue of the variability of the small blue bump, since the data examined by Maoz et al. (1993) have not been superseded by superior data and we are unlikely to be able to improve on their results. We thus concentrate on variability of the optical Fe II features, composed primarily of two blends, one between $\mathrm{H} \gamma$ and $\mathrm{H} \beta$, and the other covering the spectral range 5100-5700 $\AA$ (often referred to as the $4570 \AA$ and $5190,5320 \AA$ blends, respectively; Osterbrock 1976), and also the UV Fe II blends in the vicinity of the C IV $\lambda 1549$ emission line.

\section{METHODOLOGY AND DATA ANALYSIS}

\subsection{Data}

As noted above, we focus on NGC 5548 on account of the large amount of variability data available. There are two suitable UV data sets. The first of these is a set of IUE spectra obtained over an 8 month period in 1988-1989, sampled once every 4 days (Clavel et al. 1991). The second set of UV spectra were obtained with the Faint Object Spectrograph on the Hubble Space Telescope (HST) over a 39 day period in 1993, sampled once per day (Korista et al. 1995); this second set was supplemented by additional $I U E$ data that we disregard in this study, as they are far less suitable than the HST data for the present purposes. Both UV data sets were recovered from the International AGN Watch Web site. The IUE spectra used were those processed with the IUE New Spectral Image Processing System (NEWSIPS; Nichols et al. 1993). The HST spectra were processed as described by Korista et al. (1995).

In addition to the UV spectra, there are 1248 optical spectra that were obtained beginning in 1988 (i.e., concurrently with the IUE spectra described above) for 13 years through 2001 (Peterson et al. 2002 and references therein). However, only a relatively small subset of these data are suitable for studying variability of the optical Fe II blends; the Fe II blends are broad, low-contrast features that can be measured or modeled usefully only in highquality spectra. Also, as discussed below, we find that Fe II variability is most reliably measured by combining spectra to form difference spectra (i.e., subtraction of a low flux-state spectrum from a high flux-state spectrum) or rms spectra in order to isolate the parts of the spectrum that are varying. Since the amplitude of variability is small and since combining spectra propagates noise, it is essential to start out with high-quality data. Specifically, we require high signal-to-noise ratios, reasonably high spectral resolution in order to detect structure in the Fe II blends, and highfidelity relative flux calibration over an extended wavelength range in order to constrain the underlying continuum level. Moreover, the necessity of combining spectra requires that we select a homogeneous subset of the data. Given these requirements, the best subset is drawn from those obtained with the Lick Observatory $3 \mathrm{~m}$ telescope by A.V. Filippenko and collaborators ("Set H" described by Peterson et al. 2002 and references therein). We selected from this subset all high-quality spectra that cover the observed wavelength range 4000-6000 $\AA$. These criteria yielded 73 spectra spanning the entire 13 years of the International AGN Watch program in a fairly representative way. These 73 spectra were put on a common flux scale by assuming that the narrowline $[\mathrm{O}$ III $] \lambda \lambda 4959,5007$ fluxes are constant with $F([\mathrm{O}$ III $]) \lambda 5007=$ $5.58 \times 10^{-13} \mathrm{ergs} \mathrm{s}^{-1} \mathrm{~cm}^{-2}$ (Peterson et al. 1991). This was accomplished using a version of the van Groningen \& Wanders (1992) flux scaling algorithm. After scaling each spectrum in 


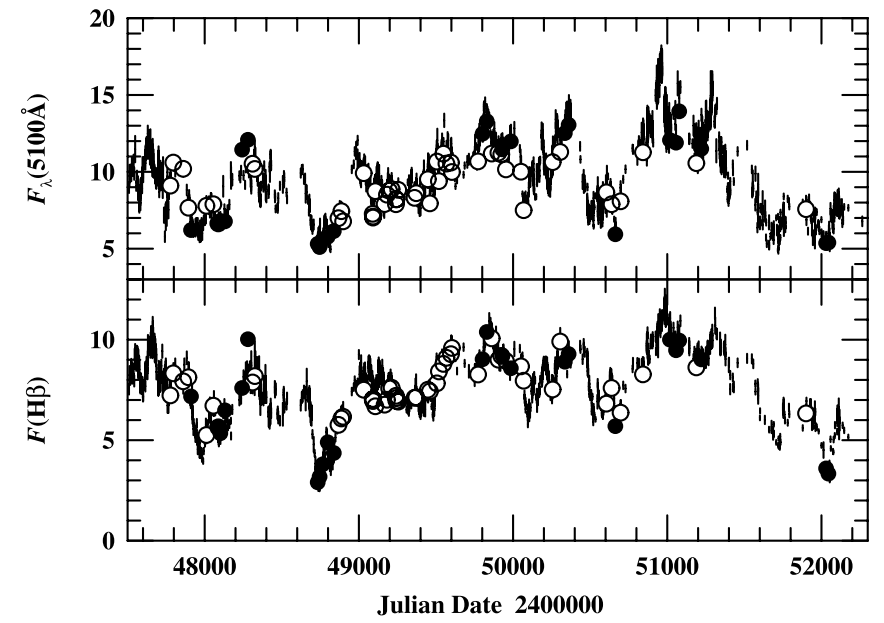

FIG. 1.-Optical continuum (top panel) and $\mathrm{H} \beta$ (bottom panel) light curves for NGC 5548 for the period 1988-2001. The vertical lines show the $\pm 1 \sigma$ error ranges for the light curves based on the International AGN Watch database (Peterson et al. 2002 and references therein), and the open and filled circles show the measurements based on the 73 rescaled spectra used in this study. The filled circles mark the epochs of the data used to construct the low- and high-state spectra shown in Fig. 4 and described in $\S 2.3 .1$. Continuum fluxes are in units of $10^{-15} \mathrm{ergs} \mathrm{s}^{-1} \mathrm{~cm}^{-2} \AA^{-1}$, and $\mathrm{H} \beta$ fluxes are in units of $10^{-13} \mathrm{ergs} \mathrm{s}^{-1} \mathrm{~cm}^{-2}$. The distribution of open and filled circles shows that this particular subset of spectra represents the entire database very well.

flux, we verified the consistency of the calibration by measuring the continuum flux at $5100 \AA$ and the total $\mathrm{H} \beta$ flux in each spectrum. Figure 1 shows a comparison of the continuum and $\mathrm{H} \beta$ fluxes measured here with published fluxes based on all of the International AGN Watch data. Agreement appears to be excellent. Figure 1 also underscores the point that the spectra we have selected for study are a suitably representative subset of the entire database.

\subsection{Fe II Templates}

As noted above, the complex atomic structure of $\mathrm{Fe}^{+}$produces a large number of transitions that pervade the UV, optical, and IR spectrum. Doppler broadening within the BLR causes the Fe II emission lines to blend together and with lines of other species, thus making it extremely difficult to accurately measure the Fe II emission-line flux. However, it was first noted by Phillips $(1977,1978)$ that the optical Fe II spectra of Seyfert 1 galaxies are very similar, differing from one AGN to the next primarily in the amount of Doppler broadening. In this context, the Seyfert 1 galaxy I Zw 1 (Sargent 1968; Phillips 1976) is of particular interest: the widths of the Fe II and Balmer lines in this source are only $\sim 1100 \mathrm{~km} \mathrm{~s}^{-1}$, among the very narrowest permitted lines observed in Seyfert 1 spectra. Phillips demonstrated that by suitably broadening the I Zw 1 spectrum to match the broad $\mathrm{H} \beta$ widths of other Seyfert 1 galaxies, the observed Fe II profiles could be matched reasonably well. This suggested that the Fe II emission arises cospatially with the broad $\mathrm{H} \beta$ emission. This also suggested to Boroson et al. (1985) and Boroson \& Green (1992) that the broadened I Zw 1 spectrum could be used as a template to model the Fe II contribution to AGN emission-line spectra, although of course their interest was more focused on removing Fe II as an emission-line contaminant than in using it to determine the Fe II emission flux. Boroson \& Green used high-quality spectra of I Zw 1 to develop an Fe II emission-line template that could be used generally to remove Fe II emission from AGN spectra over the range 4250-7000 $\AA$. Recently, a revised and improved op-

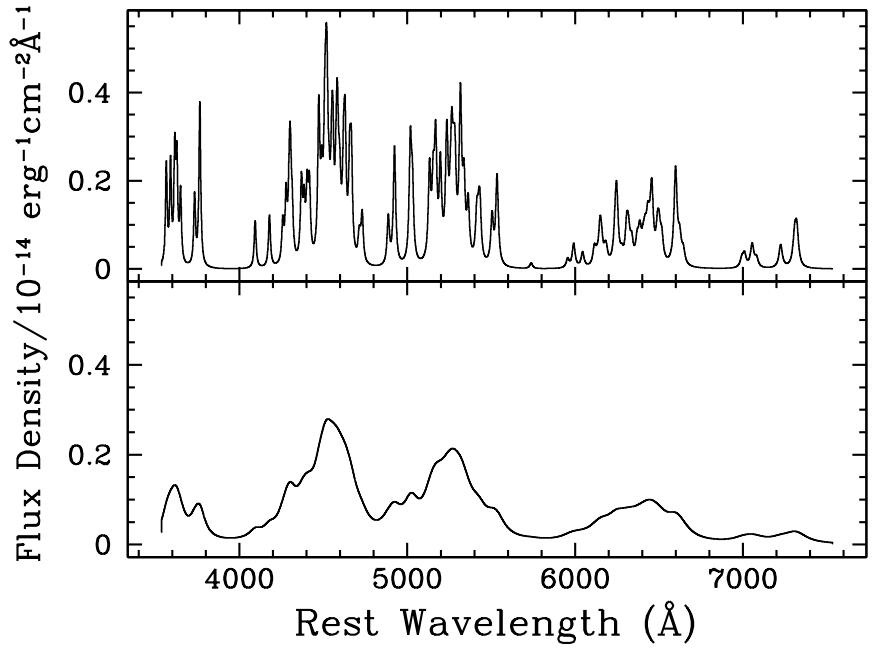

FIG. 2.-Top: Optical Fe II template constructed by Véron-Cetty et al. (2004), reproduced as described in $\S 2.2$; the intrinsic line width is $1000 \mathrm{~km} \mathrm{~s}^{-1}$ (FWHM ). Bottom: Same template, but with an intrinsic Doppler width of $6250 \mathrm{~km} \mathrm{~s}^{-1}$ (FWHM).

tical Fe II template based on I Zw 1 and covering the range 3535$7534 \AA$ A has been prepared by Véron-Cetty et al. (2004). It is this template, shown in Figure 2, that we use in analysis of the optical spectra of NGC 5548. Templates with a range of intrinsic line widths were generated by folding the entries of Fe II transitions listed in Table A.1 of Véron-Cetty et al. (their broad system L1) with Lorentzian ${ }^{4}$ profiles of different FWHMs.

A similar template for the UV spectrum (1250-3090 A), again based on I Zw 1, has been constructed from HST spectra by Vestergaard \& Wilkes (2001). We use this template, shown in Figure 3, to attempt to model the UV Fe II emission in NGC 5548. Vestergaard \& Wilkes present several versions of UV iron

\footnotetext{
4 We here follow the authors on the choice of profile shape for the template. However, the actual profile shape has little importance in this context, especially once the template is broadened.
}

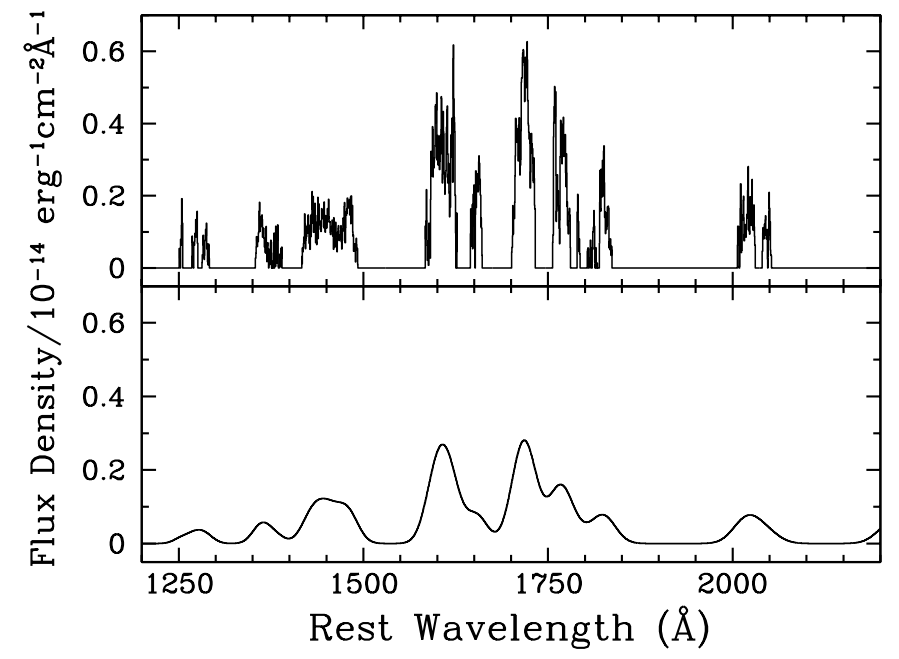

FIG. 3.-Top: Unbroadened ultraviolet Fe II template constructed by Vestergaard \& Wilkes (2001). The fluxes shortward of $1550 \AA$ are scaled by a factor of 0.25 compared to the original template of Vestergaard \& Wilkes (see $\S 2.2$ ), which yields a better fit in general to the data analyzed here. Note that no Fe III emission is present in this template. Bottom: Same template, but artificially broadened to a Doppler width of $6250 \mathrm{~km} \mathrm{~s}^{-1}$ (FWHM). 
templates, including separate templates of $\mathrm{Fe}$ II and $\mathrm{Fe}$ III emission, respectively, each of which have options of including or excluding certain multiplets that are characteristically strong in I Zw 1. Two templates of UV iron emission were fitted to the NGC 5548 data: (1) a template consisting only of Fe II emission transitions, excluding Fe II UV 191 at 1786 Å, and (2) a template consisting of both $\mathrm{Fe}$ II and $\mathrm{Fe}$ III emission transitions, excluding Fe II UV 191 and $\mathrm{Fe}$ III UV 34, as these multiplets are particularly strong in I Zw 1 and the spectra of NGC 5548 show little indication of their presence. As is made clear in $\S 2.4$, the template that includes $\mathrm{Fe}$ III emission provides a poor fit to the data. We note that the $\mathrm{Fe}$ II emission shortward of the $\mathrm{C}$ IV emission line tends to be overpredicted by using the template as originally presented by Vestergaard \& Wilkes. Forster et al. (2001) came to a similar conclusion for a small fraction of the spectra in their sample. The bluest part of the HST spectrum of I Zw 1 (below 1500 $\AA$ ) on which the UV template is based was not observed at the same time as the longer wavelength segments, and I Zw 1 increased in luminosity between these two observations. Vestergaard \& Wilkes rescaled the shortest wavelength segment to match the longer wavelength part of the spectrum to produce a full-range UV template. However, it was recognized then that the Fe II emission in the template below $\sim 1550 \AA$ may need to be rescaled for some objects. By fitting the NGC 5548 spectra we found that very reasonable fits can be obtained by applying a scaling factor of 0.25 to the template $\mathrm{Fe}$ II emission shortward of $1550 \AA$.

The general fitting procedure used here is described by Vestergaard \& Wilkes (2001, $\S 4.2$ in that paper). A power-law continuum is subtracted from the spectrum, which makes the $\mathrm{Fe}$ II emission more visible, before fitting the $\mathrm{Fe}$ II templates. The power-law continuum is fitted to windows that are virtually free of line emission, namely, $\sim 4200 \AA \AA$ and $\sim 5650 \AA \AA$ in the optical and $\sim 1350 \AA$ and $\sim 2000 \AA$ in the UV. An iteration over continuum setting and Fe II template fitting may be necessary to get the best fit. We consider the best template fit to be that which leaves the residual spectra virtually featureless in regions dominated by Fe II. Additional details on our fitting of the optical spectrum of NGC 5548 are given in $\S$ 2.3.3.

\subsection{Optical Fe II Blends}

We made an initial attempt to fit each of the 73 selected spectra with the optical $\mathrm{Fe}$ II template. We found this to be very difficult because of blending of Fe II with other features in the spectra, notably weak narrow lines and small-scale structure due to features in the underlying galaxy spectrum. Given the problems we encountered, we had little confidence in these results.

\subsubsection{Difference Spectra}

We began experimenting with alternative approaches that would mitigate some of the problems due to contaminating features. One method is to produce difference spectra, i.e., to subtract a low flux-state spectrum from a high flux-state spectrum. This effectively removes all the nonvariable components from the spectrum, including narrow emission lines and host galaxy light, although sometimes small residuals of these features can be seen, usually as a consequence of seeing and aperture effects (e.g., Peterson et al. 1995) or small differences in the line-spread function between pairs of observations. A potential liability of this approach is that the uncertainties in difference spectra can be fairly large; the differences between two spectra are often quite small, but the uncertainties add in quadrature. We attempted to mitigate this problem by making a high signal-to-noise ratio low flux-state spectrum by co-adding a number of individual faint-state spectra, and then subtracting this "standard low-state spectrum" from all of the individual spectra. To form a standard low-state spectrum, we identified several spectra obtained during the faintest periods in the 13 year monitoring program. The standard low-state spectrum as formed by averaging three spectra from the fourth year of the AGN Watch program (specifically, the spectra obtained on JD 2,448,733, 2,448,765, and 2,448,810) with two spectra obtained during the 13th year (on JD 2,452,030 and 2,452,045).

Use of difference spectra greatly improved our ability to model the optical $\mathrm{Fe}$ II emission with a template, although the results were still not quite satisfactory. We found that there was degeneracy at about the $10 \%$ level in fitting the Fe II fluxes (i.e., changes in flux of $10 \%$ made no discernible difference in the overall quality of the fit). Moreover, comparison of the inferred Fe II fluxes between observations that are closely spaced in time often showed large differences; indeed, if the errors are random, we would infer that the level of precision of the Fe II fluxes is only $\sim 30 \%$, about an order of magnitude more uncertain than our $\mathrm{H} \beta$ fluxes (Peterson et al. 2002). Despite these large uncertainties in individual spectra, we nevertheless find a clear correlation between the continuum flux at $5100 \AA$ and the Fe II flux. We find a Pearson's linear correlation coefficient of 0.73 and a probability of a chance (or null) correlation below 0.0001 . While we caution against placing too much emphasis on the specific numbers, these results emphasize the reality of such a correlation.

These measurements establish that the optical Fe II fluxes do indeed vary in a way that is correlated with the continuum. However, we are unable to establish a timescale for the response of the optical Fe II blends to continuum changes, i.e., to measure a reverberation lag. First, the sampling of these 73 spectra is comparatively sparse, as can be seen clearly in Figure 1. The median interval between observations is nearly 60 days. For reference, cross-correlation of the 73 optical continuum and $\mathrm{H} \beta$ fluxes measured from these spectra and displayed in Figure 1 yield time delays $\tau_{\text {cent }}=5.3 \pm 11.8$ days and $\tau_{\text {peak }}=17 \pm 4$ days, where these two quantities are, respectively, the centroid and peak of the optical continuum $/ \mathrm{H} \beta$ cross-correlation function; both of these measurements are consistent with the result obtained using all the AGN Watch data shown in Figure 1, although the centroid value is formally highly uncertain. Second, the very large uncertainties in the optical Fe II fluxes obviate any chance of obtaining even a marginal time-lag measurement. Formally, cross-correlation of the continuum and Fe II light curves yields $\tau_{\text {cent }}=12 \pm 27$ days and $\tau_{\text {peak }}=11 \pm 34$ days with a peak correlation coefficient $r_{\max }=0.75$. We can conclude, however, that the lag for the optical Fe II emission is less than several weeks; this is not an especially stringent upper limit, as it exceeds the value of any other reliably measured lag in NGC 5548.

Our originally planned strategy had been to start with the most suitable optical spectra and progress to the less suitable but better time-sampled data. However, we abandoned that approach when these initial results made it clear that measuring the Fe II fluxes accurately, even in the best data, is extremely difficult; measuring the Fe II fluxes in less suitable data would not improve the situation.

Inspection of the fits to our 73 difference spectra indicates that the difficulties in accurately fitting Fe II templates to the spectra are largely attributable to noise in the spectra, of both Poisson and non-Poisson origin. In other words, we might hope to obtain more accurate flux measurements by combining not only low-state but high-state spectra as well. This proved to be essential for estimating the amplitude of Fe II flux variability. In this case, we examined the continuum flux measurements for all the AGN Watch data in Figure 1 and computed their mean and 


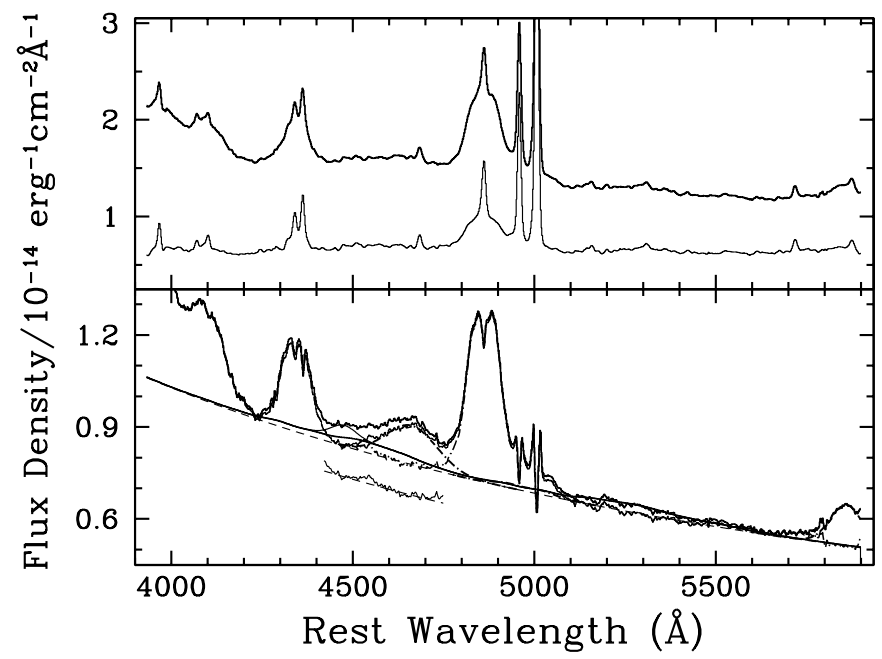

FIG. 4.-Top: High-state and low-state optical spectra of NGC 5548 in the rest frame. Bottom: Difference spectrum formed by subtraction of the low-state spectrum from the high-state spectrum (upper spectrum in the top panel) along with our preferred final fit to the spectrum, as described in $\S 2.3 .3$. Note that the residuals in the $[\mathrm{O}$ III] $\lambda \lambda 4959,5007$ lines arise from small differences in the linespread function; the total line flux in the residuals is nevertheless zero. The smooth solid thick line shows the broadened $\left(\mathrm{FWHM}=6250 \mathrm{~km} \mathrm{~s}^{-1}\right.$ ) and scaled Fe II template, the dashed line shows a fit to the underlying continuum, and the dashdotted line shows a Gaussian fit $\left(\sim 12,000 \mathrm{~km} \mathrm{~s}^{-1}\right.$ FWHM $)$ to the residual He II $\lambda 4686$ profile. The smooth solid thin line represents the He I $\lambda \lambda 4471,4922,5016$, 5876 lines; for visibility, these lines are shown on top of the Fe in template fit. The spectrum after subtraction of the Fe II template, the $\mathrm{He}_{\mathrm{I}}$ lines, and the $\mathrm{He}$ II fit is also shown (dashed line). For visibility a section of this residual spectrum and the underlying continuum is shown shifted to a lower flux level.

standard deviation. We defined a mean high-state spectrum by combining all of the spectra in our selected sample of 73 spectra for which the continuum flux is more than 1 standard deviation above the mean value. These spectra are those obtained on JD 2,448,243, 2,448,280, 2,449,802, 2,449,830, 2,449,930, $2,449,986,2,450,336,2,450,361,2,451,017,2,451,056$, $2,451,077$, and $2,451,221$. Similarly, we defined a mean lowstate spectrum by combining all of the spectra for which the continuum flux is more than 1 standard deviation below the mean values; the spectra that meet this criterion were obtained on JD 2,447,912, 2,448,089, 2,448,102, 2,448,132, 2,448,733, $2,448,746, \quad 2,448,765, \quad 2,448,798, \quad 2,448,837,2,450,664$, $2,452,030$, and $2,452,045$. We then computed the difference between these two in order to produce a high signal-to-noise ratio difference spectrum, as shown in Figures 4 and 5.

\subsubsection{Mean and rms Spectra}

Another approach to more clearly isolating the variable part of the spectrum (and thus suppressing the constant components that contaminate the optical Fe II spectrum) is to compute an rms spectrum. Using all of the 73 spectra in the sample, we formed a mean spectrum,

$$
\overline{F(\lambda)}=\frac{1}{N} \sum_{i=1}^{N} F_{i}(\lambda)
$$

where $F_{i}(\lambda)$ is the $i$ th spectrum of the $N$ spectra that comprise the database. Similarly, the rms spectrum is defined by

$$
S(\lambda)=\left\{\frac{1}{N-1} \sum_{i=1}^{N}\left[F_{i}(\lambda)-\overline{F(\lambda)}\right]^{2}\right\}^{1 / 2} .
$$

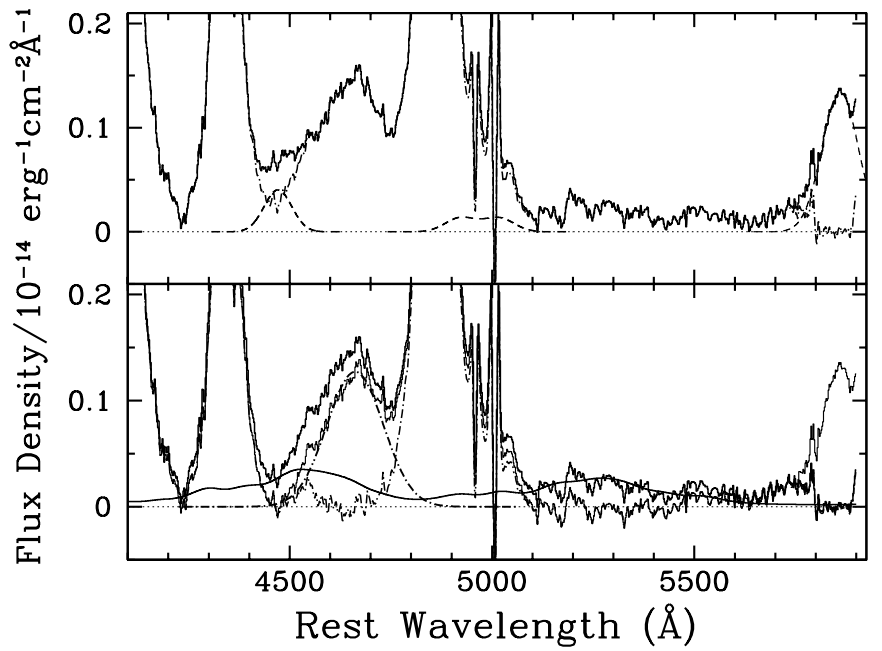

FIG. 5.-Illustration of our method for fitting the optical spectra of NGC 5548. An enlargement of the difference spectrum from the bottom panel of Fig. 4 with the continuum subtracted is shown in the top panel. The He I $\lambda \lambda 4471,4922,5016$, and 5876 lines (dashed line) and the spectrum remaining after their subtraction (dot-dashed line) are also shown. The $\lambda \lambda 4471,4922,5016$ profiles are those expected on the basis of the observed $\lambda 5876$ line (see text). In the bottom panel the smooth solid curve shows the $6250 \mathrm{~km} \mathrm{~s}^{-1}$ broadened and scaled Fe II template superposed on the continuum-subtracted and $\mathrm{He}$ I-subtracted spectrum from the top panel. The lower of the two solid histograms is the residual from subtracting this iron template fit. A simple $\left(10,500 \mathrm{~km} \mathrm{~s}^{-1}\right)$ Gaussian fit to the $\mathrm{He}$ II $\lambda 4686$ profile (dot-dashed line) and residuals with the Fe II template and He II fit subtraction is also shown. Weak residuals remain at $4543 \AA$.

The mean and rms spectra formed from our 73 optical spectra are shown in Figure 6. This mean spectrum is the only spectrum that we fitted for this discussion that does not strictly contain variable emission components only. The mean spectrum was fitted to allow assessment of the relative Fe II variability amplitude.

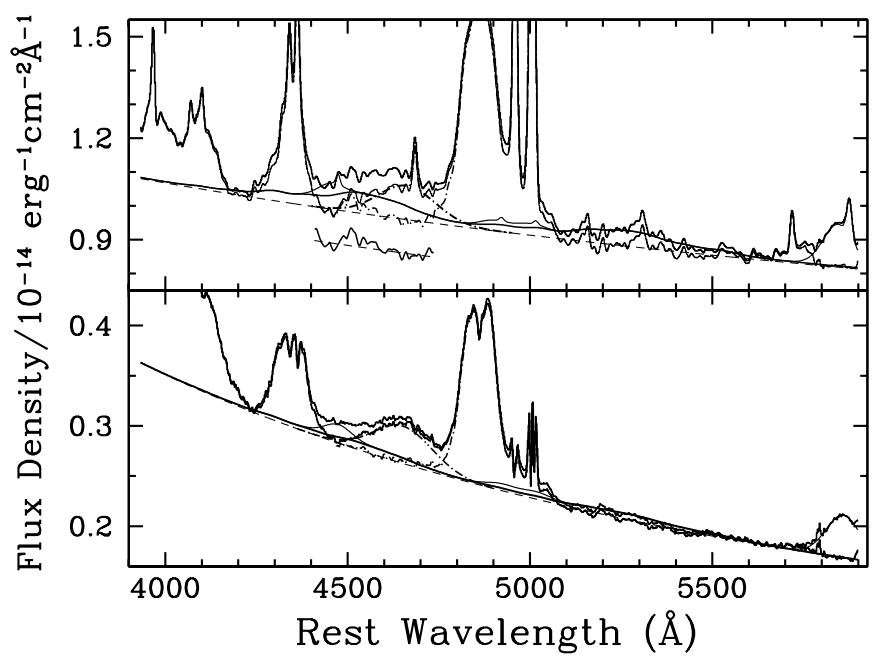

FIG. 6.-Mean (top panel) and rms (bottom panel) optical spectra of NGC 5548 in the rest frame. In both cases, the smooth solid thick line shows the broadened (6250 $\mathrm{km} \mathrm{s}^{-1}$ FWHM) and scaled Fe II template, the dashed line shows a fit to the underlying continuum, and the dash-dotted line shows a Gaussian fit to the residual He II $\lambda 4686$ profile. As in Fig. 4 , the thin solid lines atop the Fe II template fit show the four $\mathrm{He}$ I $\lambda \lambda 4471,4922,5016$, and 5876 lines. The spectrum after subtraction of the Fe II template and the He II fit is also shown. In the top panel a section of this residual is shown shifted downward for visibility. The He II profile in the mean spectrum can be fitted with two Gaussian profiles of widths $\sim 800$ and $12,000 \mathrm{~km} \mathrm{~s}^{-1}$, respectively. The width of the rms He II profile fit is $12,250 \mathrm{~km} \mathrm{~s}^{-1}$. 


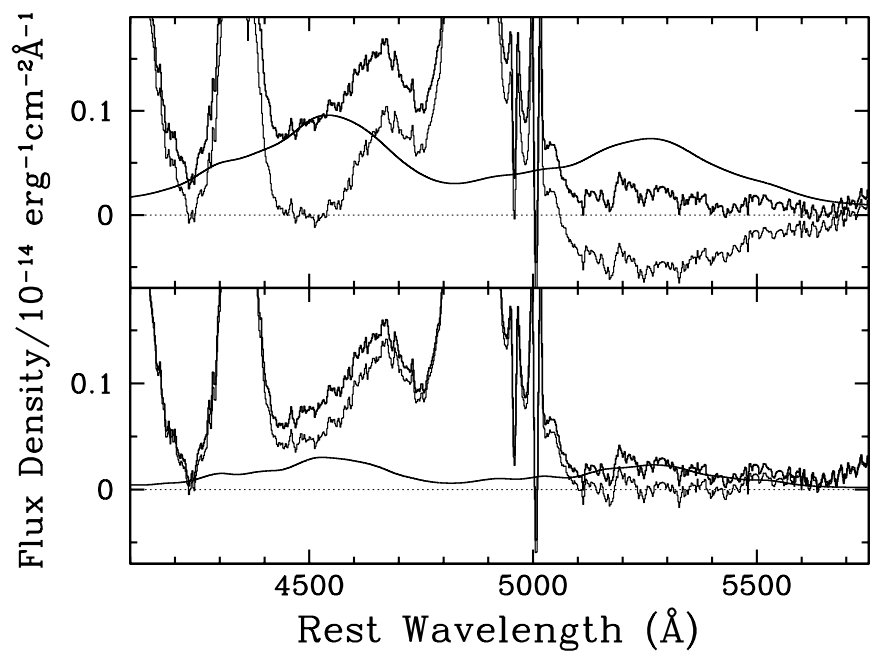

FIG. 7.- Sample fit with the Véron-Cetty et al. (2004) template to the difference spectrum shown in Fig. 5. In the top panel, the template is fitted to match the 4500 Å region only. Note the strong overprediction of the visible Fe II features between 5050 and $5550 \AA$. The bottom panel shows the template fit and residuals obtained if the template is fitted to the flux level of the $\sim 5300 \AA \mathrm{Fe}$ II transitions only. This template poorly matches the spectrum in the $4500 \AA$ region. The Fe II template has been broadened to $6250 \mathrm{~km} \mathrm{~s}^{-1}$.

\subsubsection{Identifying the Best-fitting Method of the Optical Iron Emission in NGC 5548}

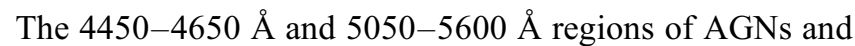
quasars are commonly dominated by Fe II emission that can be reasonably well fitted by an optical $\mathrm{Fe}$ II template generated from the I Zw 1 emission spectrum (Boroson \& Green 1992; VéronCetty et al. 2004). However, we were unable to fit these regions in the NGC 5548 optical spectra with either the Véron-Cetty et al. or the Boroson \& Green templates in their original form, i.e., with relative line intensities as observed in I Zw 1 . The top panel of Figure 7 illustrates the result of accounting for all the flux at $\sim 4500 \AA$ above a power-law continuum with the Véron-Cetty et al. Fe II template. Clearly, this strongly overpredicts the $\mathrm{Fe}$ II flux in the 5050-5700 Å range. Fitting the template to match the $5050-5600 \AA \mathrm{Fe}$ II features instead leaves strong residuals at $\sim 4500 \AA$.

We examined three possible solutions to this problem. First, the $\mathrm{Fe}$ II features may not have the relative line intensity as observed for I Zw 1 . Second, the emission at $\sim 4500 \AA$ may be due to extended wings of some combination of $\mathrm{H} \gamma, \mathrm{He}$ II $\lambda 4686$, and $\mathrm{H} \beta$. Third, additional line emission, previously unaccounted for, may be present. We address each in turn in the following.

1. We did indeed find that the spectra could be fitted well if the $5050-5600 \AA \mathrm{Fe}$ II features in the template were rescaled. The best fits to the mean, difference, and rms spectra of such modified Véron-Cetty et al. (or Boroson \& Green) templates yield scalings relative to (fixed) $\sim 4500 \AA \mathrm{Fe}$ II strength of $\sim 0.4,0.25$, and 0.2 , respectively. However, such low intensity ratios are not commonly seen in AGN spectra; relative intensities of 0.7-0.8 seem more typical. Also, it is apparently difficult to obtain very low intensity ratios of these lines in standard photoionization equilibrium modeling (M. Joly 2004, private communication). This solution is hence not particularly attractive.

2. To test whether or not extended wings of the $\mathrm{H} \gamma, \mathrm{He}$ II, and $\mathrm{H} \beta$ lines can account for the $\sim 4500 \AA$ residual emission seen in the lower panel of Figure 7 we fitted Gaussian functions to $\mathrm{H} \gamma$ and $\mathrm{He}$ II $\lambda 4686$, while a combination of Gaussian and Lorentzian

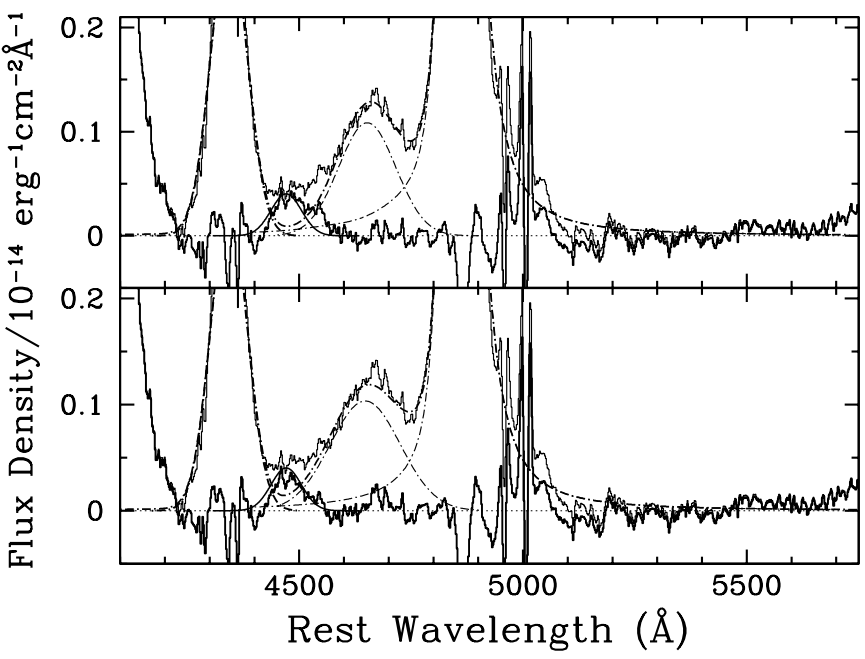

FIG. 8.-Continuum and Fe II-subtracted difference spectrum from the bottom panel of Fig. 7 shown here with the residuals (thick line) obtained when subtracting reasonable reproductions of the non-Fe profiles (dot-dashed lines). The profile fits to $\mathrm{H} \beta$ and $\mathrm{H} \gamma$ were generated especially to reproduce the blue and red sides of the core and the broad wings as allowed by the data; the details of the fits to the line peaks can be ignored, and the fit to the [O III] residuals is suggestive only. The He II $\lambda 4686$ profile is shown with a $10,000 \mathrm{~km} \mathrm{~s}^{-1}$ Gaussian profile (best fit) in the top panel, while the bottom panel shows the widest He II profile of $12,000 \mathrm{~km} \mathrm{~s}^{-1}$ allowed by the data. Neither fit can adequately account for the excess emission at $4500 \AA$. However, reasonable consistency is found with the expected strength of $\mathrm{He}$ I $\lambda 4471$ based on the observed He I $\lambda 5876$ feature. Here $\mathrm{He}_{\mathrm{I}} \lambda 4471$ is represented by a Gaussian fit to He I $\lambda 5876$, shifted to $4471 \AA$ (with fixed FWHM) and rescaled by a factor of 0.3 , a commonly established strength of $\mathrm{He}_{\mathrm{I}} \lambda 4471$. This justifies our inclusion of the He $\mathrm{I}$ lines in our fitting of this region (e.g., Figs. 4, 5, and 6).

functions was necessary to reproduce the (variable) $\mathrm{H} \beta$ profile. These modeled lines were then subtracted from the data. The results are shown in Figure 8. The upper panel shows the best-fit width to the $\mathrm{He}$ II line of $10,000 \mathrm{~km} \mathrm{~s}^{-1}$ (FWHM). A strong residual feature at $4500 \AA$ shows that we still cannot account for all the flux in this region. A second attempt with a broader $\left(12,000 \mathrm{~km} \mathrm{~s}^{-1} \mathrm{FWHM}\right) \mathrm{He}$ II line, as shown in the lower panel of Figure 8, results in very little improvement.

3. An alternative explanation is that additional previously unidentified line emission is present in the NGC 5548 spectrum. Although emission lines other than $\mathrm{Fe}$ II are rarely seen at $\sim 4500 \AA$ in quasars (e.g., Boroson \& Green 1992), NGC 5548 may have a strong contribution of $\mathrm{He}$ I $\lambda 4471$, given the presence of strong $\mathrm{He}$ I $\lambda 5876$ observed at the edge of the spectra (Figs. 4 , 5, and 6). In fact, the presence of $\mathrm{He}$ I $\lambda 5876$ also implies the existence of the He I singlet lines at 4922 and $5016 \AA$. Common intensity ratios to $\mathrm{He} \mathrm{I} 25876$ are $0.3,0.1$, and 0.1 for the 4471 , 4922, and $5016 \AA$ lines, respectively, although the $4471 \AA$ intensity ratio may be as low as 0.1 (K. Korista 2004, private communication). Both panels of Figure 8 illustrate that the excess emission at $\sim 4500 \AA$ remaining after subtracting other known sources of line emission is consistent with the presence of $\mathrm{He}$ I $\lambda 4471$ (thin solid curve) at an intensity of 0.3 times that of $\mathrm{He} \mathrm{I}$ $\lambda 5876$.

These arguments collectively justify our inclusion in our general fitting of the nonapparent $\mathrm{He}$ I $\lambda \lambda 4471,4922,5016$ lines with the aforementioned intensity ratios as found in photoionization equilibrium modeling. Figure 5 illustrates the method we thereafter adopted for fitting the Fe II emission: the expected He I features, based on the observed $5876 \AA$ line, were subtracted before fitting the $\mathrm{Fe}$ II emission. The adopted template 
fitting of the difference, mean, and rms spectra is shown in Figures 4 and 6.

We note that both the broad He I $\lambda 5876$ and He II $\lambda 4686$ features are observed shifted to the blue by $\sim 1000-1500 \mathrm{~km} \mathrm{~s}^{-1}$; this is apparent even in the observed spectra. It is particularly clear in the mean spectrum (Fig. 6), where the narrow He I $\lambda 5876$ component is observed at $5876 \AA$, yet the peak of the broad component is blueshifted; we reproduced this shift when modeling and subtracting the expected $\mathrm{He}$ I lines in the mean spectrum. Interestingly, there is no clear indication that the (broad) He II $\lambda 1640$ line is shifted by such a large amount.

Upon subtraction of the best-fit Fe II templates, a few small residuals may still remain, especially in the mean spectrum (Fig. 6) where some nonvariable possibly narrower Fe II transitions may be present; we did not attempt to include any transitions of the narrower Fe II system identified by Véron-Cetty et al. (2004), on the assumption that these would not appear in our difference or rms spectra, which appears justified by Figures 4-6. It is noteworthy that many of the smaller scale features seen atop the 4450-4750 $\AA$ emission blend in the mean spectrum are consistent with the expected positions of Fe II $\mathrm{m} 38$.

\subsubsection{Results}

Our analysis of the optical Fe II data on NGC 5548 leads us to the conclusion that the Fe II emission is indeed variable in flux and that these variations are positively correlated with continuum variations. We are unable, however, to make any meaningful determination of any time delay between the continuum and $\mathrm{Fe}$ II emission blend variations.

In an attempt to assess the amplitude of variability of the optical Fe II emission, we integrated the Fe II flux in the best-fit template over the range 4250-5710 $\AA$ and divided it by the $\mathrm{H} \beta$ broad-line flux as measured in the mean, difference, and rms spectra, respectively. The results are shown in Table 1. Comparison of the $\mathrm{Fe}$ II $/ \mathrm{H} \beta$ flux ratio in the variable spectra with the mean spectra shows that this ratio is only $25 \%-50 \%$ smaller in the variable spectra. This implies that the optical Fe II emission varies with an amplitude of $50 \%-75 \%$ that of the broad $\mathrm{H} \beta$ component. The reason that $\mathrm{Fe}$ II emission is not always obvious in rms spectra is simply because the variations are generally rather small, and the low-contrast Fe II features are weak relative to $\mathrm{H} \beta$ and do not show up very well even in high-quality difference or rms spectra.

The best fit we achieve is with an optical Fe II template broadened to a width similar to that of the variable $\mathrm{H} \beta$ line; the $\mathrm{H} \beta$ line width in the rms emission-line spectrum shown in the lower panel of Figure 6 is about $6250 \mathrm{~km} \mathrm{~s}^{-1}$. Interestingly, this template gives only a slightly improved fit to the 5050-5600 $\AA$ $\mathrm{Fe}$ II features relative to templates of somewhat broader widths of $\sim 10,000 \mathrm{~km} \mathrm{~s}^{-1}$; the differences in the fit and residuals are practically insignificant.

It is also worth noting, however, that for broadening larger than $\sim 9000 \mathrm{~km} \mathrm{~s}^{-1}$, the templates become essentially degenerate; i.e., it is hard to distinguish among them.

Given that reasonable fits are obtainable with a range of template line widths, we carried out additional tests to try to determine the maximum and minimum line widths for which the template fitting process gives unacceptable results. We find a range of template line widths that yield "acceptable" fits and a smaller range that are "preferred," for which even better fits are obtained. The degeneracy is mainly due to the flux density uncertainties in the difference spectra $(\S 2.3 .1)$ and the relatively minute differences in the detailed shapes of Fe II templates that differ by $250-750 \mathrm{~km} \mathrm{~s}^{-1}$ in line widths, especially for large line
TABLE 1

Optical Fe il/H $\beta$ Flux Ratio

\begin{tabular}{cccc}
\hline \hline $\begin{array}{c}\text { Template Width } \\
\left(\mathrm{km} \mathrm{s}^{-1}\right) \\
(1)\end{array}$ & $\begin{array}{c}\text { Mean Spectrum } \\
(2)\end{array}$ & $\begin{array}{c}\text { Difference Spectrum } \\
(3)\end{array}$ & $\begin{array}{c}\text { rms Spectrum } \\
(4)\end{array}$ \\
\hline $6250^{\mathrm{a}} \ldots \ldots \ldots \ldots \ldots \ldots \ldots \ldots \ldots$ & 0.66 & 0.48 & 0.30 \\
\hline a & 0.60
\end{tabular}

widths (above about $6000 \mathrm{~km} \mathrm{~s}^{-1}$ ). For the optical difference and rms spectra, which are not contaminated by nonvariable emission, we find that the template fits are unacceptable at line widths less than $4500 \mathrm{~km} \mathrm{~s}^{-1}$; preferred fits were obtained for widths above $5750 \mathrm{~km} \mathrm{~s}^{-1}$, and our "best-fit" value of $6250 \mathrm{~km} \mathrm{~s}^{-1}$ is a subjective assessment that this provides the best overall fit to the spectrum. Establishing an upper limit on the broadening is more difficult because of the stronger degeneracy of the templates with line widths above about $9000 \mathrm{~km} \mathrm{~s}^{-1}$. We are unable to place any meaningful constraints on the upper limit to the Fe II line widths.

Subtraction of the optical Fe II template leaves He II $\lambda 4686$ in the residual spectrum. The width of this line in the residual spectrum is very broad, $\sim 12,000 \mathrm{~km} \mathrm{~s}^{-1}$, and the width is insensitive to which Fe II template is used; i.e., the difference between using the 6250 and $10,000 \mathrm{~km} \mathrm{~s}^{-1}$ templates changes the He II $\lambda 4686$ width by less than about $200 \mathrm{~km} \mathrm{~s}^{-1}$. This He II $\lambda 4686$ line width is very similar to the width of He II $\lambda 1640$ measured in the Fe II-subtracted UV difference and rms spectra, discussed below, and to the He II $\lambda 1640$ width obtained during both UV monitoring campaigns, but is significantly larger than the width of He II $\lambda 4686$ measured in the rms spectrum, with no attempt to account for the possible effects of Fe II during the first year of the NGC 5548 monitoring campaign (Peterson et al. 2004). It is not clear, however, whether or not the He II $\lambda 4686$ width varies with time, as does, for example, the width of $\mathrm{H} \beta$, or if the base of the He II $\lambda 4686$ line gets broader when the Fe II is removed. Certainly, the responses of $\mathrm{He}_{\mathrm{II}} \lambda 1640$ and $\mathrm{He}$ II $\lambda 4686$ are expected to be identical (Bottorff et al. 2002), and the lags are, in fact, consistent within their uncertainties (Peterson et al. 2004). Indeed, there seems to be no strong evidence that either the lags or line widths of He II $\lambda 1640$ and He II $\lambda 4686$ are significantly different from one another. The concerns of Bottorff et al. regarding the differences in the response of these two lines may be not as significant as previously thought.

\subsection{UV Fe II Blends}

\subsubsection{UV Difference Spectra}

In order to determine whether or not the UV Fe II emission blends vary with the continuum, we carried out an analysis similar to that described above for the optical emission lines, although in this case we describe only our investigation of difference spectra. For both UV data sets described in $\S 2.1$, we computed mean high-state and mean low-state spectra, again using the criteria that these spectra have continuum fluxes that are 1 standard deviation above and below the mean flux, respectively. The HST high-state spectrum included those obtained on JD $2,449,121,2,449,122,2,449,123$, and 2,449,124, and those that comprise the low-state spectrum are those obtained on JD 2,449,097, 2,449,098, and 2,449,100. These spectra and their differences are shown in Figures 9 and 10. For the IUE data, the high-state spectrum was constructed from the spectra obtained on JD 2,447,538, 2,447,543, 2,447,546, 2,447,621, 2,447,625, $2,447,629, \quad 2,447,633,2,447,634, \quad 2,447,637, \quad 2,447,638$, 


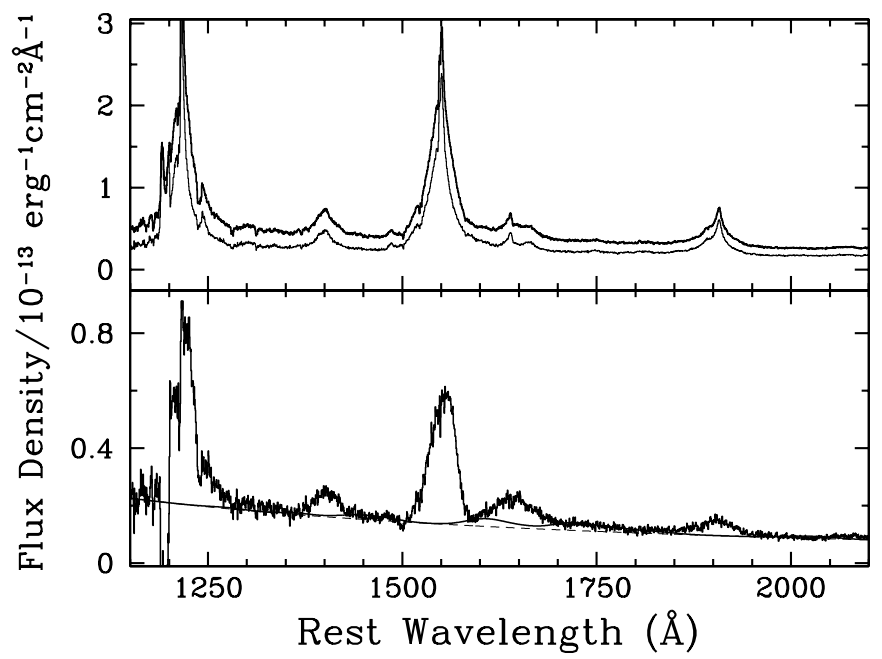

FIG. 9.-Top: High-state and low-state ultraviolet spectra of NGC 5548 in the rest frame as observed with HST in 1993. Bottom: Difference spectrum formed by subtraction of the low-state spectrum from the high-state spectrum. The smooth solid line shows the broadened (10,000 $\mathrm{km} \mathrm{s}^{-1}$ FWHM) and scaled Fe II template, and the dashed line shows a fit to the underlying continuum.

$2,447,641,2,447,645$, and 2,447,649, and the low-state spectrum is based on the spectra obtained on JD 2,447,577, $2,447,581,2,447,688,2,447,692,2,447,729,2,447,733$, $2,447,737,2,447,741$, and $2,447,745$. These spectra and their differences are shown in Figures 11 and 12.

\subsubsection{Results}

We fit the difference spectra with two types of template, (1) a pure Fe II template and (2) a template that includes both Fe II and Fe III. Both templates are from Vestergaard \& Wilkes (2001) and described in $\S 2.2$. While Fe II is clearly present in the difference spectrum, it is not clear that Fe III is present. If Fe III is present, it is very weak. We find that inclusion of Fe III is not necessary to

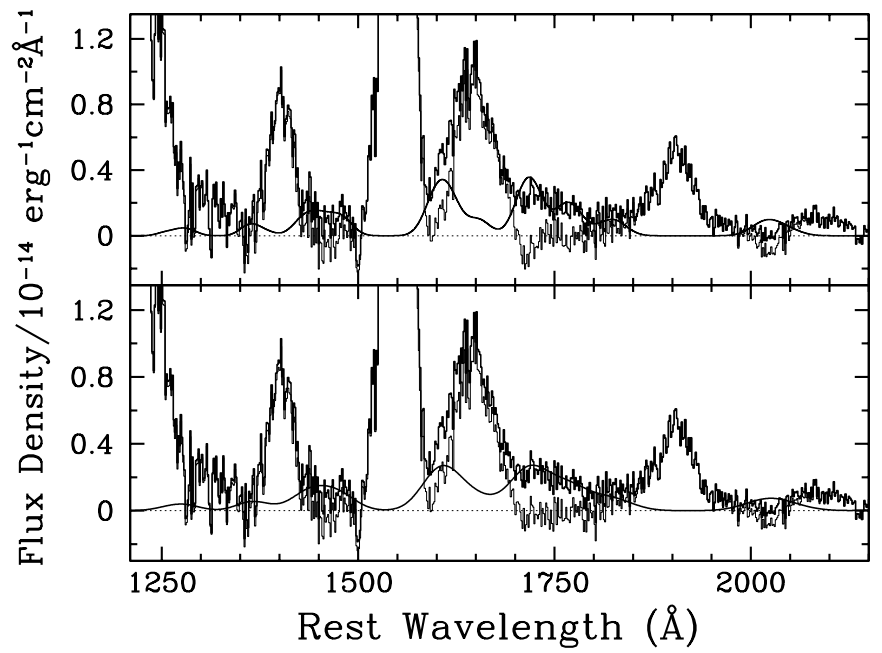

FIG. 10.-Enlargement of the difference spectrum from the bottom panel of Fig. 9 with the continuum subtracted. Again, the solid line shows the broadened and scaled Fe II template, which has a width of $6250 \mathrm{~km} \mathrm{~s}^{-1}$ in the top panel and $10,000 \mathrm{~km} \mathrm{~s}^{-1}$ in the bottom panel. Note the poor fit of the $6250 \mathrm{~km} \mathrm{~s}^{-1}$ iron template to the $1700 \AA$ region. The template overpredicts the Fe II emission at $2025 \AA$, possibly due to strong multiplets present in I Zw 1 but not in NGC 5548. The emission on either side of the expected Fe II bump at $2025 \AA$ is almost certainly Fe III (see Vestergaard \& Wilkes 2001).

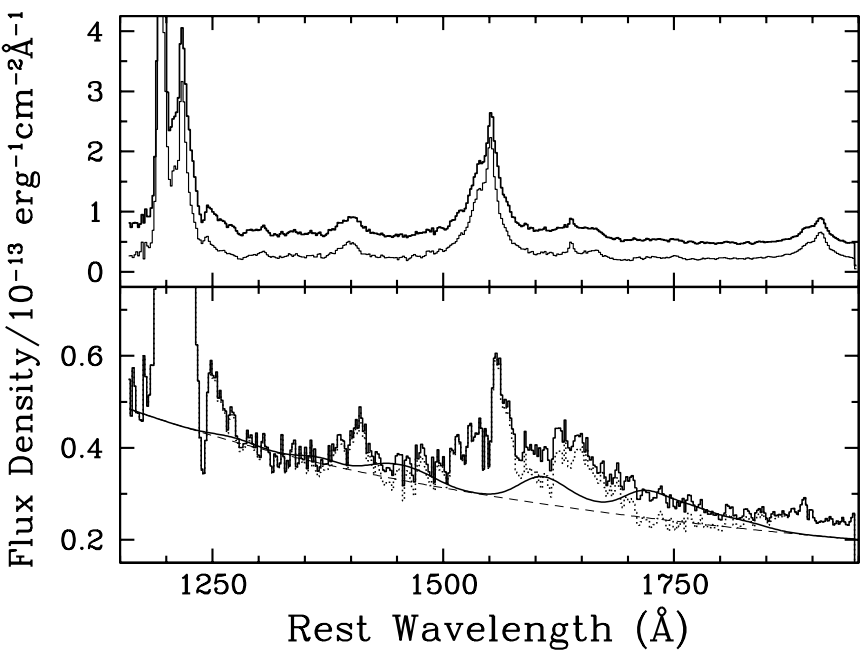

FIG. 11.-Top: High-state and low-state ultraviolet spectra of NGC 5548 in the rest frame as observed with IUE in 1988-1989. Bottom: Difference spectrum formed by subtraction of the low-state spectrum from the high-state spectrum. The smooth solid line shows the broadened $\left(10,000 \mathrm{~km} \mathrm{~s}^{-1} \mathrm{FWHM}\right)$ and scaled Fe II template, the dotted line represents the Fe II subtracted spectrum, and the dashed line shows a fit to the underlying continuum.

model the spectrum, and the spectrum is better fitted by the pure Fe II template. One possible exception is the bump at $\sim 2200 \AA$, which is most likely Fe III (Vestergaard \& Wilkes 2001). However, the results of this study are not sensitive to the strength of this feature, and we do not consider it any further. Compared to the HST spectrum, the IUE spectrum is harder to fit because it is noisier and covers a shorter wavelength range. On the other hand, if we constrain the slope of the $I U E$ difference spectrum by using the slope measured in the HST difference spectrum, we obtain a satisfactory fit. We find that a better fit is obtained with the $10,000 \mathrm{~km} \mathrm{~s}^{-1}$ template than with the $6250 \mathrm{~km} \mathrm{~s}^{-1}$ template (Figs. 10 and 12).

In particular, we find unacceptable fits using templates with broadenings less than $8250 \mathrm{~km} \mathrm{~s}^{-1}$. Given the same issues

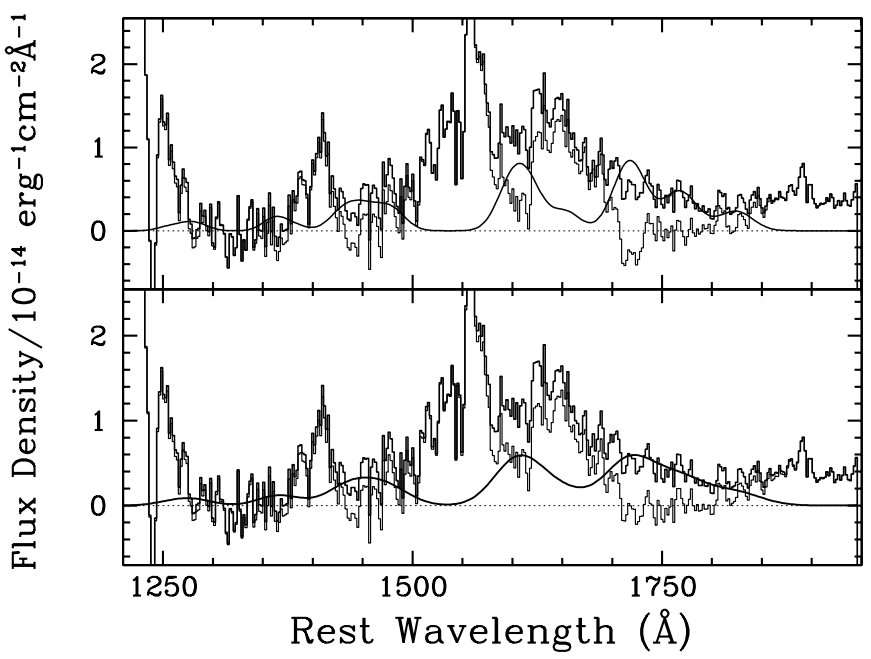

FIG. 12.-Enlargement of the difference spectrum from the bottom panel of Fig. 11 with the continuum subtracted. Again, the solid line shows the broadened and scaled Fe II template, which has a width of $6250 \mathrm{~km} \mathrm{~s}^{-1}$ in the top panel and $10,000 \mathrm{~km} \mathrm{~s}^{-1}$ in the bottom panel. As in Fig. 10, the template broadened to the rms width of broad $\mathrm{H} \beta\left(6250 \mathrm{~km} \mathrm{~s}^{-1}\right)$ yields a less satisfactory fit than that of the $10,000 \mathrm{~km} \mathrm{~s}^{-1}$ template, especially at about $1700 \AA$. The Fe II-subtracted spectra are shown as the (lower) thin-lined histograms. 
TABLE 2

Ultraviolet Fe il / H $\beta$ Flux Ratio

\begin{tabular}{|c|c|c|c|c|}
\hline $\begin{array}{c}\text { Template Width } \\
\left(\mathrm{km} \mathrm{s}^{-1}\right) \\
(1)\end{array}$ & $\begin{array}{l}H S T \text { Mean Spectrum } \\
\text { (2) }\end{array}$ & $\begin{array}{c}\text { HST Difference Spectrum } \\
\text { (3) }\end{array}$ & $\begin{array}{l}\text { IUE Mean Spectrum } \\
\text { (4) }\end{array}$ & $\begin{array}{c}\text { IUE Difference Spectrum } \\
\text { (5) }\end{array}$ \\
\hline 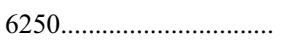 & 0.45 & 3.12 & 0.56 & 4.98 \\
\hline $10000 \ldots \ldots \ldots \ldots$ & 0.54 & 3.12 & 0.67 & 4.66 \\
\hline
\end{tabular}

pertaining to the optical spectra, we are unable to place any upper limits on template broadenings that produce acceptable fits.

We also compare the strengths of the UV Fe II blends (integrated across the two intervals $1430-1500 \AA$ and $1685-1850 \AA$ ) and $\mathrm{H} \beta$ in the variable part of the spectrum with what is observed in the mean spectrum, as we did with the optical Fe II emission above. ${ }^{5}$ The results of this comparison are given in Table 2 . We see here that the UV Fe II/H $\beta$ flux ratio is significantly larger in the variable part of the spectrum than in the mean spectrum (we note in passing that analysis of the UV mean and rms spectra leads to the same conclusion). Unlike the optical Fe II emission, the UV $\mathrm{Fe}$ II emission seems to vary with a larger amplitude than $\mathrm{H} \beta$.

\section{DISCUSSION}

The principal result of this study is that the optical and UV blends of Fe II emission in the spectrum of NGC 5548 do indeed vary with time. These variations are correlated with the continuum variations. The optical Fe II blends vary with an amplitude $50 \%-75 \%$ that of $\mathrm{H} \beta$, and the UV Fe II blends seem to vary with an even larger amplitude than $\mathrm{H} \beta$. The variations of these lowcontrast features are most clearly seen in difference or rms spectra, which remove nonvariable structure in the spectra. However, the variable $\mathrm{Fe}$ II features are not always obvious in difference or rms spectra simply because these features are often lost in the noise. Previous claims of the lack of variability of Fe II seem to be consistent with this conclusion, given the sensitivity of the observations. Unfortunately, on account of the weakness of these features in difference or rms spectra, we are unable to determine the timescale for response of the $\mathrm{Fe}$ II emission lines to continuum variations.

We note in passing that very broad Fe II features produce a "pseudo-continuum" (e.g., Wills et al. 1985) that is not readily identified as line emission. For example, Figures 9 and 11 (upper panels) show that the 1700-1900 Å region in NGC 5548 is relatively flat, giving the appearance that no (variable) emission lines are present, even though this region is at a somewhat higher level than the continuum at $\sim 1450 \AA$ and $2025 \AA$. However, the presence of Fe II emission in this spectral range is clear when fitting the spectrum with an Fe II template.

Interestingly, we find that the UV Fe II features in the difference or rms spectra are best fitted with templates that have a large velocity width, $\sim 10,000 \mathrm{~km} \mathrm{~s}^{-1}$; the optical Fe II features are consistent with both such a broad template and one of the variable $\mathrm{H} \beta$ line width. We have no ready explanation for this result, although we might speculate that it may be related to the issues responsible for the difficulty of accounting for the strength of the $\mathrm{Fe}$ II (Baldwin et al. 2004), including the unusual smoothness of the Fe II emission; either microturbulence or velocity shear seems necessary to account for the discrepancy between theory and observation.

\footnotetext{
${ }^{5}$ The $\mathrm{H} \beta$ fluxes were measured in mean or difference spectra constructed from optical spectra that are contemporaneous with the UV spectra used.
}

The issue of whether or not the optical and UV Fe II is emitted from the same region is important for theoretical modeling of their relative emission strengths (Baldwin et al. 2004). While the errors on the Fe II emission-line response time are very large, it is possible to constrain the size of the Fe II-emitting regions indirectly through the line-broadening parameters under the assumption that $\mathrm{Fe}$ II follows the same virial relationship seen in every other well-characterized variable line in NGC 5548 (Peterson \& Wandel 1999). If we assume that the virial product $c \tau_{\text {cent }} \mathrm{FWHM}^{2} / G$ is the same for all lines in NGC 5548, using measurements from Peterson et al. (2004), we can conclude that the optical Fe II emission, with FWHM $>4500 \mathrm{~km} \mathrm{~s}^{-1}$, arises at $r<24$ lt-days, and the UV Fe II emission, with FWHM $>$ $8500 \mathrm{~km} \mathrm{~s}^{-1}$, arises at $r<7$ lt-days. This limit on the size of the optical Fe II emission gas does not violate the weak constraint on the time delay of several weeks based on cross-correlation of the continuum and optical Fe II emission light curves ( $§ 2.3 .1$ ). Given the assumptions, this is a weak conclusion, but it is worth noting that differing line widths for the UV and optical Fe II emission suggest that the UV and optical Fe II might be emitted predominantly from different regions within the BLR. If indeed the BLR gas density increases steadily and smoothly toward the center of the BLR, the distance limits are broadly consistent with expectations from photoionization models (e.g., Fig. 11 of Baldwin et al. 2004), namely, that UV Fe II is most efficiently emitted from higher density gas (presumably located closer to the central source), while optical Fe II is more efficiently emitted from lower density gas farther out.

Véron-Cetty et al. (2004) find that the I Zw 1 Fe II emission spectrum is composed of two components of different widths, $\sim 1100$ and $\sim 300 \mathrm{~km} \mathrm{~s}^{-1}$, the latter mostly being forbidden iron transitions. This suggests that some of the Fe II emission originates in the narrow-line region. The fact that no variations are detected in the narrow $\mathrm{Fe}$ II features by virtue of their absence in the residual spectra of the difference and rms spectra is consistent with this explanation; blends of narrow Fe II lines may be present in the mean optical spectrum, especially longward of [O III]. We cannot identify narrow Fe II in any of the UV spectra we have examined.

Our conclusion that the amplitude of optical Fe II variability is somewhat smaller than that of $\mathrm{H} \beta$ but is often of insufficient contrast for reliable detection seems consistent with results for other sources (Kollatschny et al. 1981; Kollatschny \& Fricke 1985; Giannuzzo \& Stirpe 1996; Doroshenko et al. 1999). Apparent exceptions are Fairall 9 and NGC 7603. Kollatschny et al. (2000) found the optical $\mathrm{Fe}$ II to vary as strongly as $\mathrm{H} \beta$ for NGC 7603. In the case of Fairall 9, Kollatschny \& Fricke find that both $\mathrm{H} \beta$ and the optical Fe II emission vary with large amplitudes, by factors of 5.6 and 2.6, respectively. For more direct comparison with this result, we restricted our Fe II measurement window to the 4570, 4928, and $5018 \AA$ multiplets, the approximate region used by Kollatschny \& Fricke, and found values similar to those we quote in Table 1 . We conclude that the relative amplitudes of $\mathrm{Fe}$ II and $\mathrm{H} \beta$ flux variability can differ significantly among AGNs. 
As current photoionization models fall short in reproducing the observed AGN Fe II emission, Collin \& Joly (2000) suggest that $\mathrm{Fe}$ II in AGNs is due entirely to shock (i.e., collisional) excitation. They draw parallels with strong Fe II-emitting stellar sources that have no X-ray or ionizing emission but do have strong variability and strong outflows. Baldwin et al. (2004) do find that collisional excitation can explain the observed Fe II, although they are reluctant to consider the introduction of another component, which produces no other significant emission, into an already complex picture of the BLR. Moreover, it is not clear how such a model could reproduce the observed correlation between the optical Fe II and the continuum fluxes that we observe.

The template fits show that the I Zw 1 Fe II templates are generally reasonably good matches to the optical and UV Fe II emission from NGC 5548. The exceptions are, as noted earlier, the apparent absence of the Fe III transitions over the range 1825-2100 $\AA$ and the poor match of the original templates at $2025 \AA$, where the Fe II emission appears to be missing.

\section{CONCLUSIONS}

Our chief conclusions of our study of the Fe II emission in NGC 5548 can be summarized as follows:

1. The optical and UV Fe II emission blends do indeed vary in response to variations in the continuum emission, i.e., the Fe II and continuum flux variations are strongly correlated. Detection of these variations is difficult because these lines are very broad and of low contrast.

2. The optical Fe II varies in strength with an amplitude of $50 \%-75 \%$ that of $\mathrm{H} \beta$. The UV Fe II varies much more strongly.

3. These results are consistent with most earlier studies claiming no variability owing to the difficulty of detecting small-scale variations in low-contrast features.

4. Unfortunately, the weakness of the Fe II features precludes obtaining a meaningful variability timescale of this emission. The flux variations of the optical Fe II blends lag behind those in the continuum by no more than several weeks.
5. For reasons that are unclear, we find somewhat improved fits using UV Fe II templates with large widths of the order of $10,000 \mathrm{~km} \mathrm{~s}^{-1}$, as opposed to templates with widths similar to that of $\mathrm{H} \beta$.

6. Fe II removal improves measurement of other emission lines. The He II $\lambda 4686$ feature in the iron-subtracted spectrum displays a line width consistent with that of He II $\lambda 1640$, as expected from photoionization models. Failure to account for the effect of Fe II emission seems to yield a He II $\lambda 4686$ line width that is too small.

7. Because we cannot meaningfully constrain either the line width or the reverberation response timescale for Fe II, we are unable to conclusively determine whether or not the Fe II emission originates in the same region as $\mathrm{H} \beta$ or whether the UV and optical Fe II are spatially coemitted; this issue has importance for the theoretical modeling of the relative UV and optical Fe II emission strengths (e.g., Baldwin et al. 2004).

However, the line widths below which the optical and UV templates produce unacceptable fits to the observed $\mathrm{Fe}$ II emission do suggest that the UV and optical Fe II may originate in different regions, although there may be some overlap of these regions given our inability to place meaningful upper limits on the Fe II line widths.

The authors are grateful for support of this research by NASA through grant HST-AR-09549-01.A from the Space Telescope Science Institute, which is operated by the Association of Universities for Research in Astronomy, Inc., under NASA contract NAS5-26555, and by NSF through grant AST 0205964. Kirk T. Korista, Monique Joly, Mira Véron-Cetty, and Philippe Véron are thanked for helpful discussions and comments on an earlier version of the manuscript. We wish to thank Kirk Korista in particular for pointing out the possible importance of the He I lines. Mike Goad is thanked for a helpful and constructive referee's report.

\section{REFERENCES}

Baldwin, J. A., Ferland, G. J., Korista, K. T., Hamann, F., \& LaCluyzé, A. 2004, ApJ, 615, 610

Blandford, R. D., \& McKee, C. F. 1982, ApJ, 255, 419

Boroson, T. A. 1989, ApJ, 343, L9

Boroson, T. A., \& Green, R. F. 1992, ApJS, 80, 109

Boroson, T. A., Persson, S. E., \& Oke, J. B. 1985, ApJ, 293, 120

Bottorff, M. C., Baldwin, J. A., Ferland, G. J., Ferguson, J. W., \& Korista, K. T. 2002, ApJ, 581, 932

Clavel, J., et al. 1991, ApJ, 366, 64

Collin, S., \& Joly, M. 2000, NewA Rev., 44, 531

Davidson, K., \& Netzer, H. 1979, Rev. Mod. Phys., 51, 715

Dietrich, M., Hamann, F., Appenzeller, I., \& Vestergaard, M. 2003, ApJ, 596,817

Doroshenko, V. T., Sergeev, S. G., Pronik, V. I., \& Chuvaev, K. K. 1999, Astron. Lett., 25, 569

Forster, K., Green, P. J., Aldcroft, T. L., Vestergaard, M., Foltz, C. B., \& Hewett, P. C. 2001, ApJS, 134, 35

Giannuzzo, M. E., \& Stirpe, G. M. 1996, A\&A, 314, 419

Goad, M. R., Koratkar, A. P., Axon, D. J., Korista, K. T., \& O’Brien, P. T. 1999, ApJ, 512, L95

Hamann, F., \& Ferland, G. 1999, ARA\&A, 37, 487

Kollatschny, W., Bischoff, K., \& Dietrich, M. 2000, A\&A, 361, 901

Kollatschny, W., \& Fricke, K. 1985, A\&A, 146, L11

Kollatschny, W., Fricke, K., Schleicher, H., \& Yorke, H. W. 1981, A\&A, 102, L23

Kollatschny, W., \& Welsh, W. F. 2001, in Probing the Physics of Active Galactic Nuclei by Multiwavelength Monitoring, ed. B. M. Peterson, R. S. Polidan, \& R. W. Pogge (San Francisco: ASP), 449

Korista, K. T., et al. 1995, ApJS, 97, 285
Maoz, D., et al. 1993, ApJ, 404, 576

Nichols, J. S., Garhart, M. P., De La Peña, M. D., \& Levay, K. L. 1993, in International Ultraviolet Explorer New Spectral Image Processing System Informational Manual: Low-Dispersion Data, Version 1.0, CSC/SD-93/6062 (Greenbelt: NASA)

Osterbrock, D. E. 1976, ApJ, 203, 329

Peterson, B. M. 1993, PASP, 105, 247

2001, in Advanced Lectures on the Starburst-AGN Connection, ed. I. Aretxaga, D. Kunth, \& Raúl Mújica. (Singapore: World Scientific), 3

Peterson, B. M., Pogge, R. W., Wanders, I., Smith, S. M., \& Romanishin, W. 1995, PASP, 107, 579

Peterson, B. M., \& Wandel, A. 1999, ApJ, 521, L95

Peterson, B. M., et al. 1991, ApJ, 368, 119 1999, ApJ, 510, 659 2002, ApJ, 581, 197 2004, ApJ, 613, 682

Phillips, M. M. 1976, ApJ, 208, 37

. 1977, ApJ, 215, 746

. 1978, ApJS, 38, 187

Salamanca, I., Alloin, D., \& Pelat, D. 1995, A\&AS, 111, 283

Sargent, W. L. W. 1968, ApJ, 152, L31

Sergeev, S. G., Pronik, V. I., Malkov, Yu, F., \& Chuvaev, K. K. 1997, A\&A, 320,405

Shastri, P., Wilkes, B. J., Elvis, M., \& McDowell, J. C. 1994, ApJ, 431, 901

Sigut, T. A. A., \& Pradhan, A. K. 2003, ApJS, 145, 15

van Groningen, E., \& Wanders, I. 1992, PASP, 104, 700

Verner, E., Bruhweiler, F.,Verner, D., Johansson, S., Kallman, T., \& Gull, T. 2004, ApJ, 611, 780 
Verner, E. M., Verner, D. A., Korista, K. T., Ferguson, J. W., Hamann, F., \& Ferland, G. J. 1999, ApJS, 120, 101

Véron-Cetty, M.-P., Joly, M., \& Véron, P. 2004, A\&A, 417, 515

Vestergaard, M., \& Wilkes, B. J. 2001, ApJS, 134, 1

Wamsteker, W., et al. 1990, ApJ, 354, 446
Wilkes, B. J., Elvis, M., \& McHardy, I. 1987, ApJ, 321, L23

Wilkes, B. J., Kuraszkiewicz, J., Green, P. J., Mathur, S., \& McDowell, J. C. 1999, ApJ, 513, 76

Wills, B. J., Netzer, H., \& Wills, D. 1985, ApJ, 288, 94

Zheng, W., \& O’Brien, P. T. 1990, ApJ, 353, 433 